01

\title{
Метод расчета спектра показателей Ляпунова для систем с запаздыванием
}

\author{
() А.Д. Колоскова, О.И. Москаленко "А.А. Короновский
}

Саратовский национальный исследовательский государственный университет им. Н.Г. Чернышевского, Саратов, Россия

ฯ E-mail: o.i.moskalenko@gmail.com

Поступило в Редакцию 28 декабря 2017 г.

Предложен метод расчета спектра показателей Ляпунова для систем с запаздыванием. Для апробации метода в качестве модельных систем рассмотрены генератор с запаздыванием и уравнение Маккея-Гласса. Для обеих систем построены бифуркационные диаграммы и спектры показателей Ляпунова в зависимости от одного из управляющих параметров. Показано, что полученные результаты находятся в хорошем согласии друг с другом, что свидетельствует о работоспособности предложенного метода.

DOI: 10.21883/PJTF.2018.09.46061.17167

Дифференциальные уравнения с отклоняющимся аргументом получили широкое распространение, в частности, в иммунологии [1], химии [2], электронике [3], механике деформируемого твердого тела [4] и термодинамике [5]. Введение запаздывания в дифференциальные уравнения в ряде случаев позволяет корректно описать процессы, протекающие в рассматриваемых системах. Так, в задаче о динамике популяций оно может учитывать характеристики их развития, рождаемости или вымирания [6], в биологических задачах в качестве времени запаздывания используется время транспорта молекул от места их синтеза к месту их включения в реакцию, время формирования клеток определенного типа, участвующих в иммунной реакции [6]. В радиофизике в связи с ростом интереса к применению сложных режимов генерации СВЧ электронных приборов используются автогенераторы с запаздывающей связью $[7,8]$.

При изучении подобных систем необходимо использовать различные методы и подходы. Наглядное представление о свойствах системы можно получить с помощью временни́х реализаций, фазовых портретов, 
бифуркационных диаграмм, фурье- и вейвлет-спектров и т.п. (см., например, $[9,10])$. Одним из важных способов изучения динамики является расчет спектра показателей Ляпунова, который снабжает исследователей более детальной информацией о природе наблюдаемых режимов и поведении системы при изменении управляющих параметров $[11,12]$.

Системы с запаздыванием характеризуются бесконечномерным фазовым пространством, и соответственно спектр показателей Ляпунова является счетным, т.е. содержит бесконечное число показателей. Ранее для пространственно распределенных динамических систем, которые характеризуются также фазовым пространством с бесконечной размерностью, был предложен метод, позволяющий найти спектр показателей Ляпунова $[11,13]$, основанный на рассмотрении эволюции во времени пространственного состояния изучаемой системы и (с периодическими ортогонализациями и перенормировками) его возмущений. Несмотря на то что системы с запаздыванием также являются динамическими системами с бесконечномерным фазовым пространством, использовать для них метод, разработанный для пространственно распределенных систем, не представляется возможным, однако идеологию подхода, примененного для пространственно распределенных систем, можно взять за основу для разработки метода расчета спектра показателей Ляпунова для систем с отклоняющимся аргументом.

В настоящей работе предложен метод расчета спектра показателей Ляпунова для систем с запаздыванием. В качестве исследуемой модели было выбрано уравнение генератора с запаздыванием [14]

$$
\frac{d x(t)}{d t}=-x(t)+k F(x(t-\tau)),
$$

где $x$ - переменная, характеризующая поведение системы, $\tau$ - время запаздывания, $F(x)=a-x^{2}, a, k-$ управляющие параметры, а также рассмотрена модель кроветворения Маккея-Гласса [15]

$$
\frac{d x(t)}{d t}=\beta \frac{x(t-\tau)}{1+x^{n}(t-\tau)}-\gamma x(t),
$$

где $x(t)$ - число клеток (эритроцитов) в момент времени $t ; \tau-$ время запаздывания; $n, \beta, \gamma$ - управляющие параметры ( $n$ - целое число). Рассматриваемые системы имеют разную природу, что свидетельствует о широкой области применения предложенного метода.

Письма в ЖТФ, 2018, том 44, вып. 9 
Основная идея метода заключается в следующем. Для расчета спектра показателей Ляпунова используются состояние системы и ортогонализованный набор возмущений этого состояния, при этом каждое возмущение соответствует одному из показателей в спектре. В случае рассматриваемых одномерных систем это скалярные величины, определенные на интервале времени с длительностью, равной времени запаздывания, при этом верхняя граница используемого интервала совпадает с текущим моментом времени. В начальный момент времени необходимо выбрать возмущения таким образом, чтобы они были ортогональными

$$
\left(\tilde{x}_{i}(\xi), \tilde{x}_{j}(\xi)\right)= \begin{cases}1, & i=j \\ 0, & i \neq j\end{cases}
$$

и характеризовались единичной нормой

$$
\left\|\tilde{x}_{i}(\xi)\right\|=1
$$

Такой набор функций возмущений опорного состояния нетрудно получить при помощи процедуры ортогонализации Грама-Шмидта и последующих перенормировок [16]. При нахождении нормы (3) и осуществлении процедур ортогонализации и перенормировки используется скалярное произведение возмущений

$$
\left(\tilde{x}_{i}(t), \tilde{x}_{j}(t)\right)=\int_{t-\tau}^{t} \tilde{x}_{i}(\xi), \tilde{x}_{j}(\xi) d \xi,
$$

норма находится в виде

$$
\left\|\tilde{x}_{i}(\xi)\right\|=\sqrt{\left(\tilde{x}_{i}(\zeta), \tilde{x}_{i}(\zeta)\right)}
$$

Первым этапом при нахождении показателей Ляпунова является численное решение уравнения с запаздывающим аргументом, описывающего динамику системы, на достаточном интервале времени. Таким образом становится известной эволюция во времени опорного состояния, относительно которого рассматривается набор возмущений. Одновременно осуществляется расчет эволюции набора возмущений

Письма в ЖТФ, 2018, том 44, вып. 9 

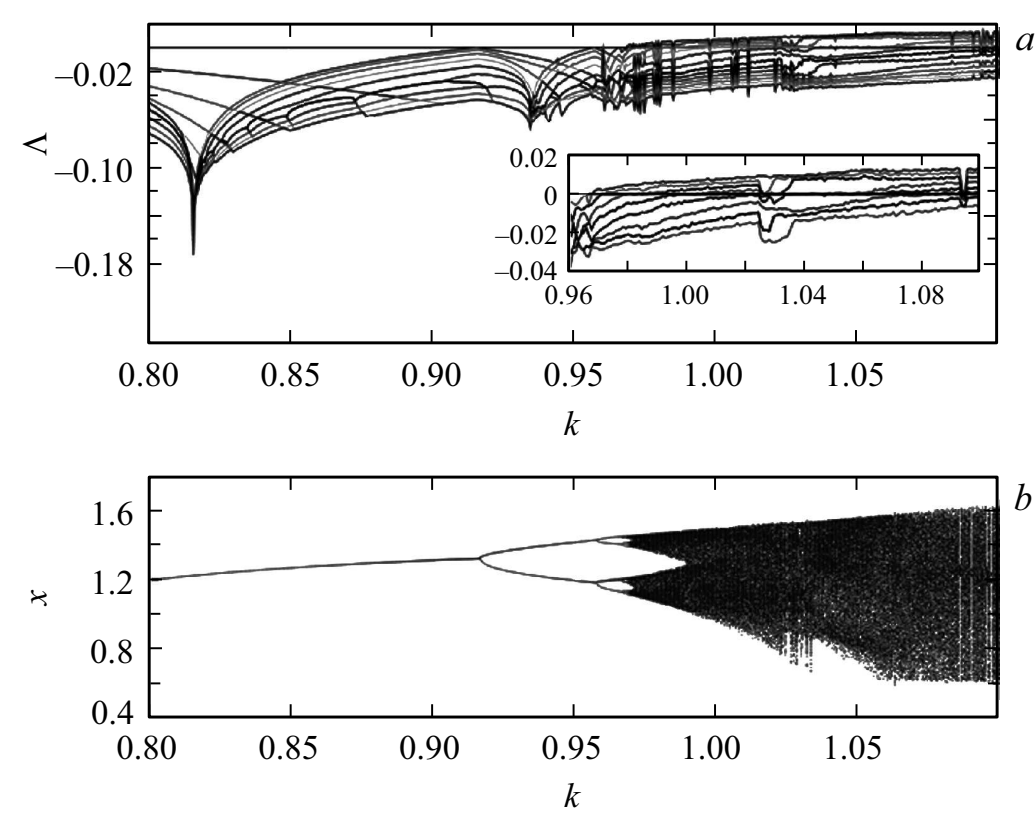

Рис. 1. Зависимости девяти старших показателей Ляпунова от параметра $k$ $(a)$ и бифуркационная диаграмма $(b)$ автогенератора с запаздыванием (1) при $a=1.5, \tau=20$.

опорного состояния системы, для чего численно интегрируются линеаризованные уравнения для каждого возмущения опорного состояния. Применительно к рассматриваемым модельным системам это

$$
\frac{d \tilde{x}(t)}{d t}=-\tilde{x}(t)+k F^{\prime}(x(t-\tau)) \tilde{x}(t-\tau)
$$

и

$$
\frac{d \tilde{x}(t)}{d t}=\beta \frac{1-(n-1) x^{n}(t-\tau)}{\left(1+x^{n}(t-\tau)\right)^{2}} \tilde{x}(t-\tau)-\gamma \tilde{x}(t)
$$

для генератора с запаздыванием (1) и уравнения Маккея-Гласса (2) соответственно.

На следующем этапе отслеживается эволюция всех рассматриваемых величин. Через равные промежутки времени вновь осуществляется 

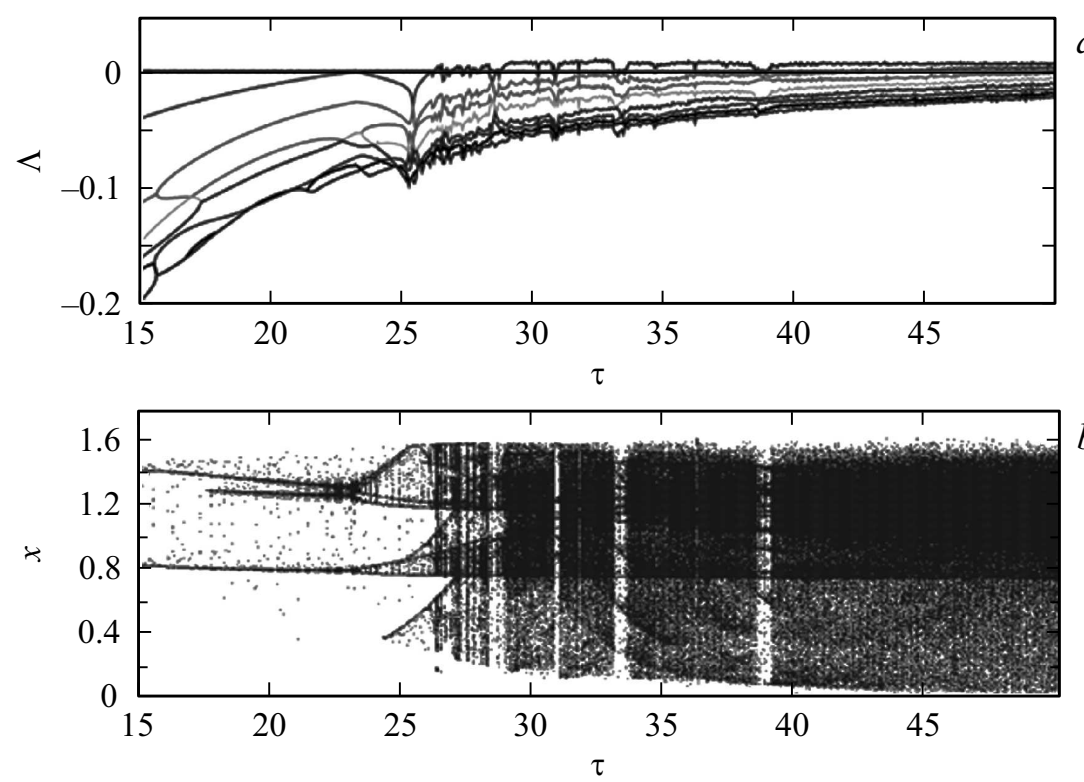

Рис. 2. Зависимости девяти старших показателей Ляпунова от времени запаздывания $\tau(a)$ и бифуркационная диаграмма $(b)$ для уравнения Маккея-Гласса (2) при $n=21, \beta=0.2, \gamma=0.1$.

ортогонализация Грама-Шмидта возмущений опорного состояния и для каждого возмущения вычисляется слагаемое ляпуновской суммы

$$
S_{i}(M \Gamma)=\sum_{k=0}^{M} \ln \|\tilde{x}(\xi)\|, \xi \in(k \Gamma-\tau, k \Gamma),
$$

где $\Gamma$ - интервал времени между применением процедур ортогонализации и нормировки, $\Gamma>\tau$. Наконец, для получения единичной нормы всех возмущений осуществляется перенормировка. Данная последовательность процедур повторяется многократно ( $M$ раз), и на каждом шаге считаются суммы для каждого возмущения до перенормировки и после ортогонализации. Промежуточные значения показателей Ляпунова оцениваются как

$$
\hat{\Lambda}_{i}(M \Gamma)=\frac{S_{i}(M \Gamma)}{M \Gamma}
$$

Письма в ЖТФ, 2018, том 44, вып. 9 
После многочисленного повтора всех описанных выше операций $(M \gg 1)$, когда значения (4) практически перестанут менять свою величину, процедуру вычислений спектра значений показателей Ляпунова

$$
\Lambda_{i}=\lim _{M \rightarrow \infty} \hat{\Lambda}_{i}(M \Gamma)
$$

для системы с запаздыванием можно завершить. В итоге набор возмущений опорного состояния системы оказывается упорядоченным по величине соответствующих им показателей Ляпунова: первое возмущение соответствует самому старшему показателю Ляпунова, второе второму по старшинству показателю и т.д. Очевидно, для получения желаемого количества показателей Ляпунова в спектре необходимо рассматривать такое же количество возмущений.

Результаты применения предложенного метода к рассматриваемым системам - генератору с запаздыванием (1) и уравнению Маккея-Гласса (2) - иллюстрируют рис. 1 и 2 соответственно. Для обеих систем был проведен расчет девяти старших показателей Ляпунова $(a)$ и построены бифуркационные диаграммы $(b)$. Полученные зависимости сопоставлены друг с другом. Из рисунков видно, что для обеих рассматриваемых систем в выбранном диапазоне изменений управляющих параметров (параметр $k$ для генератора с запаздыванием, время запаздывания $\tau$ для уравнения Маккея-Гласса) имеет место каскад бифуркаций удвоения периода с последующим переходом к хаосу при $k \approx 0.96$ и $\tau \approx 26$ соответственно (см. также [17]). При данных значениях управляющих параметров старший показатель Ляпунова переходит в область положительных значений. В области периодических режимов старший показатель Ляпунова равен нулю, а в точках бифуркаций второй показатель Ляпунова обращается в нуль в полном соответствии с результатами теоретических предсказаний.

Таким образом, в настоящей работе предложен метод расчета спектра показателей Ляпунова для систем с запаздыванием. На примере двух модельных систем (автогенератора с запаздыванием и уравнения Маккея-Гласса) показана корректность метода, что следует из хорошего согласия динамических режимов на бифуркационных диаграммах исследуемых систем и сигнатуры рассчитанного для них спектра показателей Ляпунова. Можно ожидать, что предложенный метод будет оставаться работоспособным для других более сложных динамических систем, описываемых уравнениями с запаздывающим аргументом.

Письма в ЖТФ, 2018, том 44, вып. 9 
Работа выполнена при поддержке Российского научного фонда (проект № 14-12-00224).

\section{Список литературы}

[1] Белых Л.Н., Асаченков А.Л. // Вычислительные процессы и системы. М.: Наука, 1985. В. 3. С. 12-79.

[2] Янушевский P.T. Управление объектами с запаздыванием. М.: Наука, 1978. $410 \mathrm{c}$.

[3] Теодорчик К.Ф. Автоколебательные системы. М.-Л.: Гостехиздат, 1952. $272 \mathrm{c}$.

[4] Работнов Ю.Н. Элементы наследственной механики твердых тел. М.: Наука, 1977. $384 \mathrm{c.}$

[5] Дэй У.А. Термодинамика простых сред с памятью. М.: Мир, 1974. $192 \mathrm{c}$.

[6] Долгий Ю.Ф., Сурков П.Г. Математические модели динамических систем с запаздыванием. Екатеринбург: Изд-во Урал. ун-та, 2012. 122 с.

[7] Рыскин Н.М., Шигаев А.М. // ЖТФ. 2002. Т. 72. В. 7. С. 1-8.

[8] Рыскин Н.М., Шигаев А.М. // ЖТФ. 2006. Т. 76. В. 1. С. 72-81.

[9] Божокин С.В., Суслова И.М. // ЖТФ. 2013. Т. 83. В. 12. С. 26-32.

[10] Павлов А.Н., Храмов А.Е., Короновский А.А., Ситникова Е.Ю., Макаров В.А., Овчинников А.А. // УФН. 2012. Т. 182. № 9. С. 905-939.

[11] Hramov A.E., Koronovskii A.A., Maksimenko V.A., Moskalenko O.I. // Phys. Plasmas. 2012. V. 19. N 8. P. 082302.

[12] Максименко В.А., Постнов Д.Е., Короновский А.А., Макаров В.В., Храмов A.E. // Письма в ЖТФ. 2017. Т. 43. В. 12. С. 96-103.

[13] Koronovskii A.A., Hramov A.E., Maksimenko V.A., Moskalenko O.I., Alekseev K.N., Greenaway M.T., Fromhold T.M., Balanov A.G. // Phys. Rev. B. 2013. V. 88. P. 165304.

[14] Пономаренко В.И., Прохоров М.Д. // Письма в ЖТФ. 2002. Т. 28. В. 16. C. 37-44.

[15] Mackey M.C., Glass L. // Science. 1977. V. 197. N 4300. P. 287-289.

[16] Кузнецов С.П. Динамический хаос. М.: Физматлит, 2006. 286 с.

[17] Junges L., Gallas J.A.C. // Phys. Lett. A. 2012. V. 376. N 30-31. P. 2109-2116.

Письма в ЖТФ, 2018, том 44, вып. 9 